\title{
Transcriptome analysis indicated that Salmonella lipopolysaccharide-induced thymocyte death and thymic atrophy were related to TLR4-FOS/JUN pathway in chicks
}

Haibo Huang ${ }^{1}$, An Liu ${ }^{2}$, Hui Wu ${ }^{2}$, Abdur Rahman Ansari ${ }^{1}$, Jixiang Wang ${ }^{1}$, Xiyao Huang ${ }^{1}$, Xing Zhao ${ }^{1}$, Kemei Peng ${ }^{1}$, Juming Zhong ${ }^{1,3}$ and Huazhen Liu ${ }^{1 *}$

\begin{abstract}
Background: Thymus is the crucial site for T cell development and once believed to be immune privileged. Recently, thymus has gained special attention as it is commonly targeted by infectious agents which may cause pathogenic tolerance and subsequent immunosuppression.

Results: We analyzed thymic responses to the challenge with Salmonella typhimurium (STm) or lipopolysaccharide (LPS) derived from STm in chicks. Newly hatched chicks were injected intraperitoneally with $5 \times 10^{4} \mathrm{CFU} / \mathrm{mL} \mathrm{STm}$ or $50 \mathrm{mg} / \mathrm{kg}$ LPS. After LPS treatment, maximum thymocyte death ( $3 \sim 5$-fold change) compared to controls was found at $12 \mathrm{~h}$, and maximum loss of thymic weight (35\%) and reduced thymic index (20\%) were found at $36 \mathrm{~h}$. After STm infection, maximum thymocyte death and thymic atrophy occurred at 36 and 72 h, respectively. No significant changes of thymic structure, chT1+ and CD4+/CD8+ T cell ratio were observed in thymus or spleen tissues after LPS treatment. Furthermore, transcriptome analysis revealed important roles for the TLR4-FOS/JUN signaling pathway in thymic injury. Thus, the major process of thymic atrophy in this study first involved activation of transcriptional factors FOS/JUN upon LPS binding to TLR4 that caused release of inflammatory factors, thereby inducing inflammatory responses and DNA damage and ultimately cell cycle arrest and thymic injury.
\end{abstract}

Conclusions: STm and Salmonella LPS could induce acute chick thymic injury. LPS treatment acted faster than STm. TLR4-FOS/JUN pathway may play an important role in LPS induced chick thymic injury.

Keywords: Salmonella typhimurium, Thymus injury, chT1, Transcriptome

\section{Background}

The thymus is the primary immune organ providing naïve $\mathrm{T}$ cells for peripheral immune tissues [1]. Theoretically, thymic injury can cause serious consequences, which are related to local tissue homeostasis and immune development, especially in young individuals with an immature immune system. Due to the existence of the blood-thymus barrier, the thymus was once deemed to be immune privileged [2]. However, in mammals, multiple pathogens can target the thymus and cause

\footnotetext{
* Correspondence: Ihz219@mail.hzau.edu.cn

${ }^{1}$ Department of Basic Veterinary Medicine, College of Animal Science and Veterinary Medicine, Huazhong Agricultural University, Wuhan 430070, China

Full list of author information is available at the end of the article
}

thymic injury, including extensive cell death, tissue structure abnormality, organ atrophy and functional disorder, including recent thymic emigrant abnormality and T cell tolerance to pathogens [3, 4]. In birds, pathogens including viruses (e.g., chicken anemia virus, Marek's disease virus and avian leukosis virus), bacteria (e.g., Escherichia coli) and parasites (e.g., Ascaridia galli) can also induce thymic atrophy [5-9]. Therefore, thymus injury also may be a common occurrence during infection in birds.

Salmonella typhimurium (STm) is one of the most deleterious food-borne pathogens that can induce typhoid fever and enteritis through infected chicken eggs and meat [10]. STm infection could also cause the death of newly hatched chicks [11]. STm infection has 
been reported to induce thymic atrophy, thymocyte death and a modest decrease of recent $\mathrm{T}$ cell export in mice $[12,13]$. Moreover, one study indicated that the thymic injury induced by STm in mice was mediated by intracellular JNK signaling and its downstream effectors, including reactive oxygen species (ROS) and inflammatory cytokines (e.g., TNF- $\alpha$ and IFN- $\gamma$ ) [13]. In chickens, several studies have indicated that STm infection can induce expression of chemokines and cytokines (e.g., IL-8 and IL-1 $\beta$ ) in the spleen, liver and intestinal tissues, which may contribute to the host defense against STm $[14,15]$. However, unlike in mammals, few studies have reported the impact of STm infection on the thymus in birds, and the molecular mechanism of chicken thymus response to STm infection is largely unknown.

In the present study, we evaluated the thymic injury and potential mechanisms induced by both STm and Salmonella LPS in newly hatched chicks. We found that both STm and LPS stimulation could induce acute thymus injury in chicks. The effects of LPS are faster than that of STm. Moreover, we found that the TLR4FOS/JUN signaling pathway may play a key role in this process. Our results offer novel evidence for the molecular mechanism of the thymus injury induced by Salmonella LPS.

\section{Results}

\section{Thymic atrophy after LPS treatment}

Newly hatched chicks were injected i.p. with $5 \times$ $10^{4} \mathrm{CFU} / \mathrm{mL} \mathrm{STm}$ or $75 \%$ saline (control) for the challenge of STm, and with $50 \mathrm{mg} / \mathrm{kg}$ Salmonella LPS or $75 \%$ saline (control) for the challenge of LPS. The thymic weight was assessed at $0 \sim 120 \mathrm{~h}$ post treatment (hpt). Compared with saline treatment, treatment with LPS derived from STm resulted in the maximum $35 \%$ loss of thymus weight $(P<0.01,0.18 \pm 0.04 \mathrm{~g}$ vs. $0.27 \pm$ $0.05 \mathrm{~g})$ and $20 \%$ decrease of thymus index $(P<0.05$, $2.66 \pm 0.37$ vs. $3.34 \pm 0.40)$ at $36 \mathrm{hpt}$; meanwhile, it caused $17 \%$ loss of thymus weight $(P<0.05,0.30 \pm$ $0.05 \mathrm{~g}$ vs. $0.36 \pm 0.03 \mathrm{~g})$ and $17 \%$ loss of thymus index $(P<0.05,2.81 \pm 0.23$ vs. $3.37 \pm 0.38)$ at 72 hpt (Fig. 1a and $\mathrm{b})$. The thymus weight was restored to the normal level at 120 hpt (Fig. 1a and b). Moreover, there is a dosage effect of LPS on thymic weight $(P<0.05)$ and index $(P<0.05)$ (Fig. 1c and d). Compared with saline treatment, STm infection resulted in the maximum $43 \%$ loss of thymus weight $(P<0.01,0.13 \pm 0.02 \mathrm{~g}$ vs. $0.22 \pm 0.05 \mathrm{~g})$ and $26 \%$ decrease of thymus index $(P<0.05,1.86 \pm 0.25$ vs. $2.52 \pm 0.55)$ at $72 \mathrm{hpt}$ compared with controls, and these effects were attenuated at $120 \mathrm{hpt}$ (Additional file 1: Figure S1). Furthermore, the thymus maintained structural integrity with distinct boundaries of the medulla and cortex at different stages of LPS treatment (Fig. 1e).
Therefore, both STm and Salmonella LPS treatment induced acute thymic atrophy, but the maximum LPSinduced thymic atrophy occurred $36 \mathrm{~h}$ earlier than that caused by STm infection.

\section{Thymocyte death after LPS treatment}

We assessed thymocyte death using two classic methods, the formamide-MAb assay, which recognizes damaged single-strand DNA in early apoptotic cells [16-18], and the TUNEL assay, which detects low-molecular-weight damaged DNA fragments in late apoptotic cells or necrotic cells $[17,19]$. These damaged DNA fragments are abundant in apoptotic cells [16-19]. The formamideMAb assay revealed that the maximum LPS-induced thymocyte death occurred at $12 \mathrm{hpt}$, which was 3-fold higher than that of the control $(P<0.001,40 \pm 11.00$ vs. $14 \pm 5.65$ per $10^{5} \mu \mathrm{m}$ ). Additionally, the positive cells were about 2 -fold higher at 36 and 72 hpt. These effects have also been detected by the TUNEL assay. The results of TUNEL assay were almost identical to that of the formamide-MAb assay, and the TUNEL positive cells were about 5 -fold higher than that of the control at 12 hpt (Fig. 2). During STm infection, the maximum rate of thymocyte death occurred at $36 \mathrm{~h}$, and the positive cells were 4-fold higher than that of control $(P<$ $0.01,40 \pm 15.96$ vs. $11 \pm 1.57$ per $\left.10^{5} \mu \mathrm{m}\right)$. They were attenuated by 2 -fold $(P<0.001,19 \pm 2.44$ vs. $10 \pm 1.07$ per $\left.10^{5} \mu \mathrm{m}\right)$ at $72 \mathrm{hpt}$ as detected by the formamide-MAb assay (Additional file 1: Figure S1). Therefore, both STm and Salmonella LPS treatment induced thymocyte death, and the maximum death of thymocytes occurred $24 \sim 36 \mathrm{~h}$ ahead of the peak organic atrophy. Moreover, LPS-induced thymocyte death occurred $24 \mathrm{~h}$ earlier than that stimulated by STm infection (Fig. 2; Additional file 1: Figure S1), which coincided with the phenomenon of LPS-mediated thymus atrophy ahead of that induced by STm infection (Fig. 1; Additional file 1: Figure S1). In all cases, thymocyte death was mainly located in the thymic cortex.

\section{Maintenance of chT1+ and CD4+/CD8+ cell ratios at $36 \mathrm{~h}$ after LPS treatment}

In order to analyze changes in cell sub-populations during LPS stimulation, we detected chT1+, CD4+ and CD8+ T cells in the spleen and CD4+ and CD8+ T cells in the thymus at $36 \mathrm{~h}$ after LPS treatment using flow cytometry. The development of $\mathrm{T}$ cells in thymus undergoes stages of CD4-CD8- double negative (DN) cells, CD4 + CD8+ double positive (DP) cells and CD4 + CD8- or CD4-CD8+ single positive (SP) cells [1]. T cells migrated from thymus to the periphery express chT1 for a short time and chT1+ is typically used to assess recent thymic export [20-22]. Under LPS stimulation, the proportion of chT1+ cells in spleen tissue was 

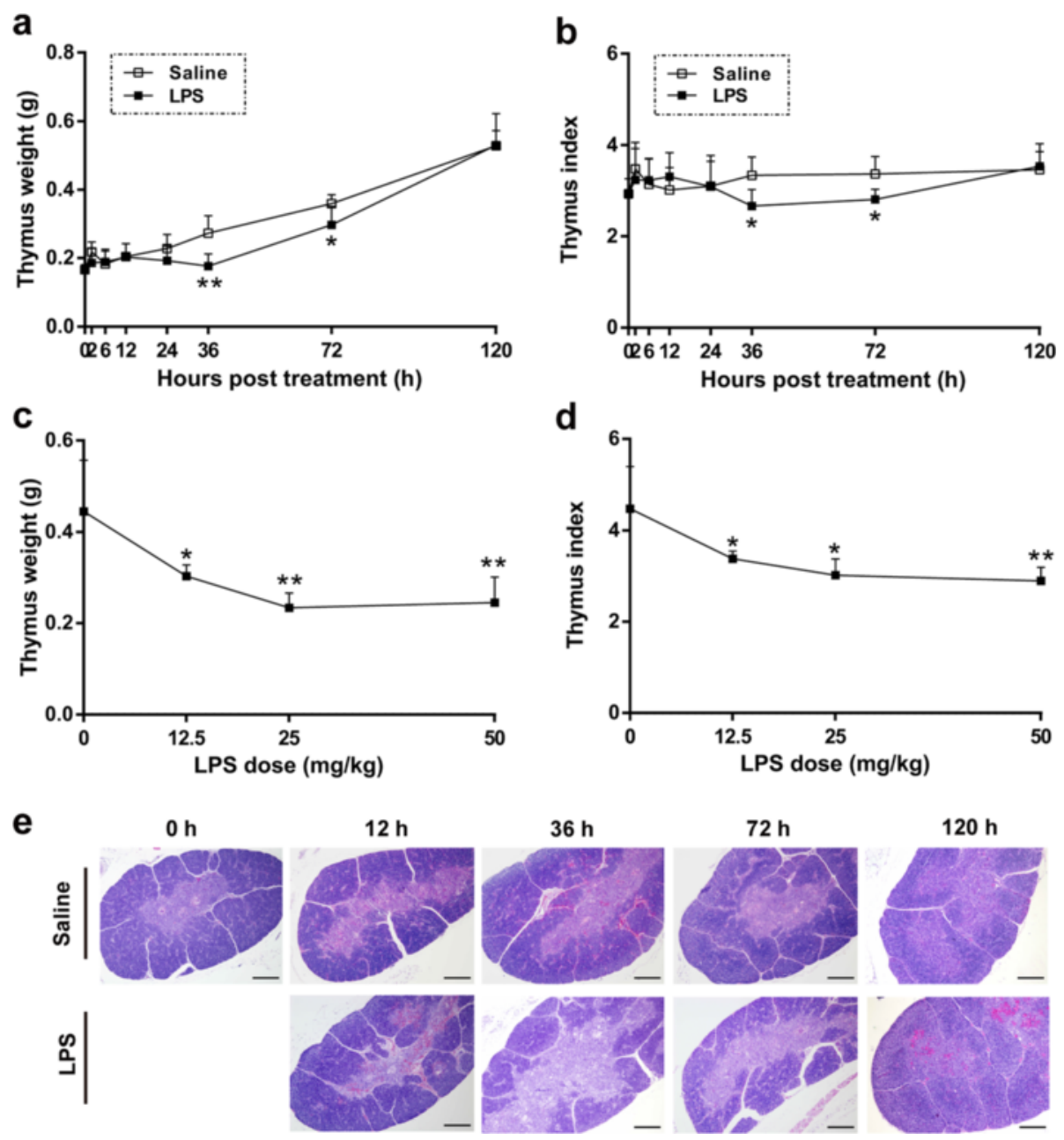

Fig. 1 Salmonella LPS induced acute thymic atrophy in chicks. Newly hatched chicks were injected i.p. with saline or Salmonella LPS and then sacrificed at defined time points to analyze thymus weight and index. $\mathbf{a}, \mathbf{b}$ LPS treatment $(50 \mathrm{mg} / \mathrm{kg}$ ) reduced thymus weight (a) and index (b) in chicks $(n=4 \sim 6)$ at 36 and 72 hpt. Statistically significant differences between LPS and saline groups at each time point were determined using Student's t-test. (c, d) LPS treatment decreased chick thymic weight (c) and index (d) in a dose-dependent manner $(n=3 \sim 5)$ at 36 hpt. Statistically significant differences between multiple LPS dose groups versus control group $(0 \mathrm{mg} / \mathrm{kg}$ LPS) was performed with Bonferroni's multiple comparisons test after one-way ANOVA test. e Sections of thymuses from LPS ( $50 \mathrm{mg} / \mathrm{kg}$ ) or saline treated chicks were stained with hematoxylin and eosin to analyze changes of tissue structure. Light areas represent the medulla, and dark areas represent the cortex. Scale bars $=200 \mu \mathrm{m}$. All data are presented as means \pm SD. ${ }^{*} P<0.05,{ }^{* *} P<0.01$

not significantly different from that of the saline control group in this study. Proportions of DP and SP T cells also showed no significant difference in the spleen and thymus (Fig. 3). Therefore, both chT1 cell ratios in spleen and CD4+/CD8+ T cell ratios in thymus or spleen were not significantly changed at $36 \mathrm{~h}$ after LPS treatment.

\section{Identification of DETs in thymus after LPS challenge}

To determine the molecular mechanism of the effects induced by LPS treatment, we detected gene expression profiles in the thymus at $0,12,36$ and 72 hpt using the RNA-seq method. In total, 76,769,194 clean reads were mapped to 27,391 transcripts. In order to identify the differential expressed transcripts (DETs), transcript expression levels at 12, 36 and $72 \mathrm{hpt}$ were compared with those at $0 \mathrm{hpt}$. As a result, 873 DETs (507 up-expressed and 366 down-expressed) were identified at $12 \mathrm{hpt}, 536$ DETs (419 up-expressed and 117 down-expressed) at 36 hpt and 856 DETs (556 up-expressed and 300 downexpressed) at $72 \mathrm{hpt}$ (Fig 4a; Additional file 2: Table S1) (absolute fold-change $\geq 1.5, P<0.001$ ). We found the maximum number of DETs at $12 \mathrm{hpt}$ and the minimum number of DETs at $36 \mathrm{hpt}$. Many of the top $10 \mathrm{up}$ - and down-expressed transcripts were related to immune responses, including AVD, IL8L2, IL4I1 and IL22 (Fig 4b; Additional file 2: Table S1). 


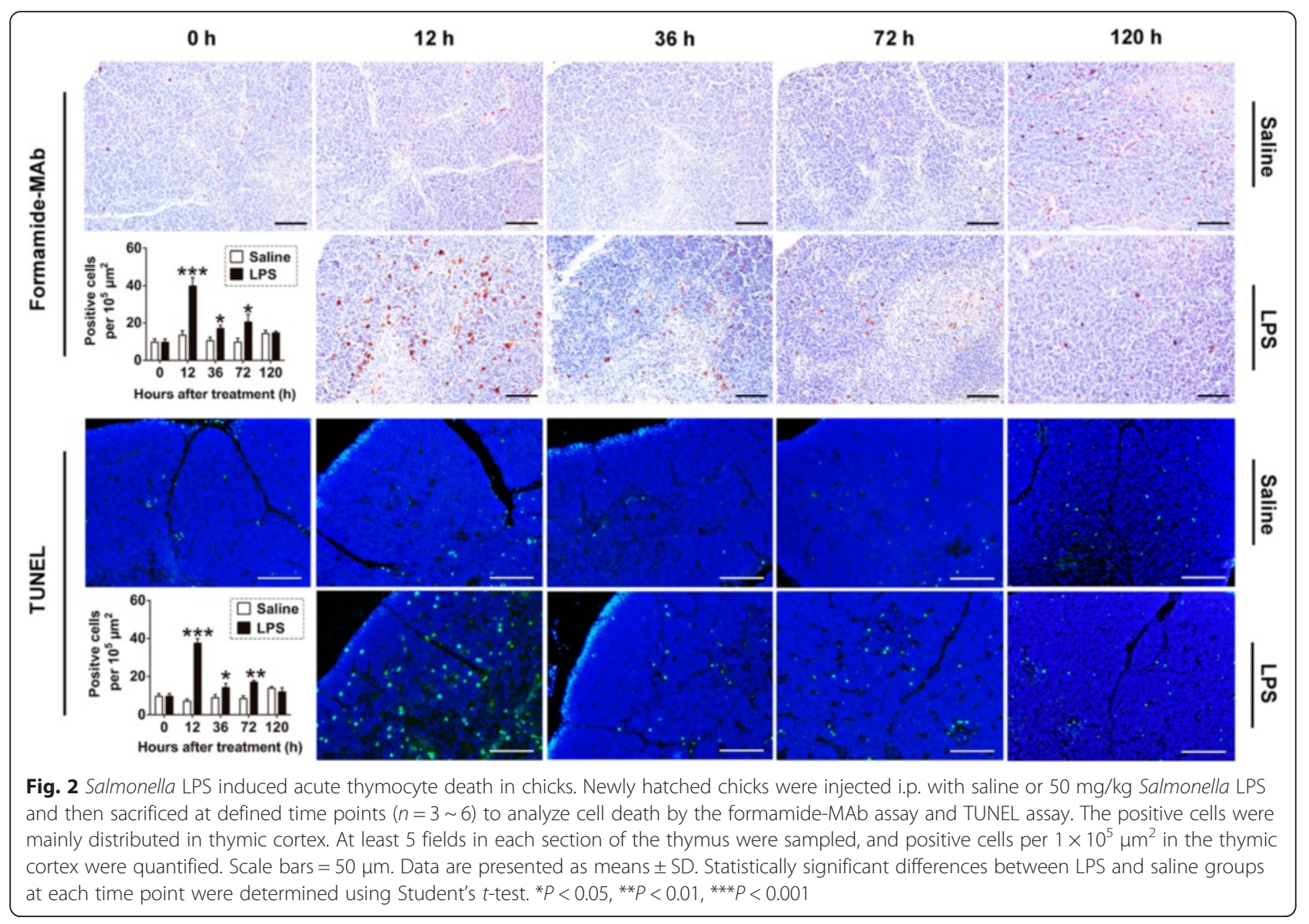

The expression patterns of ten genes in chick thymus were detected by qPCR

To validate the results of RNA-seq, ten up-expressed genes, including TLR4, TLR15, AVD, IL8L2, BPI, SOCS3, IL6ST, IL1R2, HSPB1 and NOV, were chosen for qPCR detection. In order to accurately describe the expression patterns of genes, thymic tissue RNA at eight time points, including $0,2,6,12,24,36,72$ and $120 \mathrm{~h}$ after LPS challenge, were collected for qPCR detection. The results showed that the gene expression patterns detected by qPCR were similar to those of obtained by the RNA-seq method (Fig. 5). There was a significant correlation between the changes of genes detected by RNAseq and qPCR methods (Additional file 1: Figure S2). This finding confirmed the reliability of the RNA-seq data. Based on the qPCR results, all ten genes were upexpressed at $12 \sim 24 \mathrm{~h}$ after LPS treatment. Moreover, the TLR15, AVD, IL8L2, BPI, SOCS3 and IL1R2 genes were up-expressed as early as $2 \mathrm{~h}$ after LPS treatment, and most of them were restored to the normal level after $36 \mathrm{~h}$ of LPS treatment (Fig. 5). Similarly, all of these ten genes were up-expressed with STm infection. However, the peak of the gene expression under STm infection occurred later than that in the LPS-treated group (Additional file 1: Figure S3). This result indicated that the change in expression of genes during STm infection occurred later relative to that with LPS treatment. The difference in gene expression between LPS treatment and STm infection was consistent with the observed differences in thymocyte death and thymic atrophy between those two conditions. All of the results indicated that these biological events occurred later with STm infection than with LPS stimulation.

\section{TLR4-FOS/JUN signaling pathway may mediate LPS-induced thymic atrophy}

IPA was performed on DETs to identify the key signaling pathways triggered by LPS. We found that immunerelated signaling pathways, including the complement system, granulocyte adhesion and diapedesis pathway, IL-6 signaling pathway and the acute phase response signaling pathway, were activated at 12 and $36 \mathrm{~h}$ post LPS treatment. Moreover, we found that cell cycle-related signaling pathways were down-regulated at $12 \mathrm{~h}$ post LPS treatment (Fig. 6a; Additional file 3: Table S2). We further analyzed the genes involved in the acute phase response signaling and cell cycle related pathways at 12 and 36 h. Genes, including TLR4, FOS, JUN, IL22, 

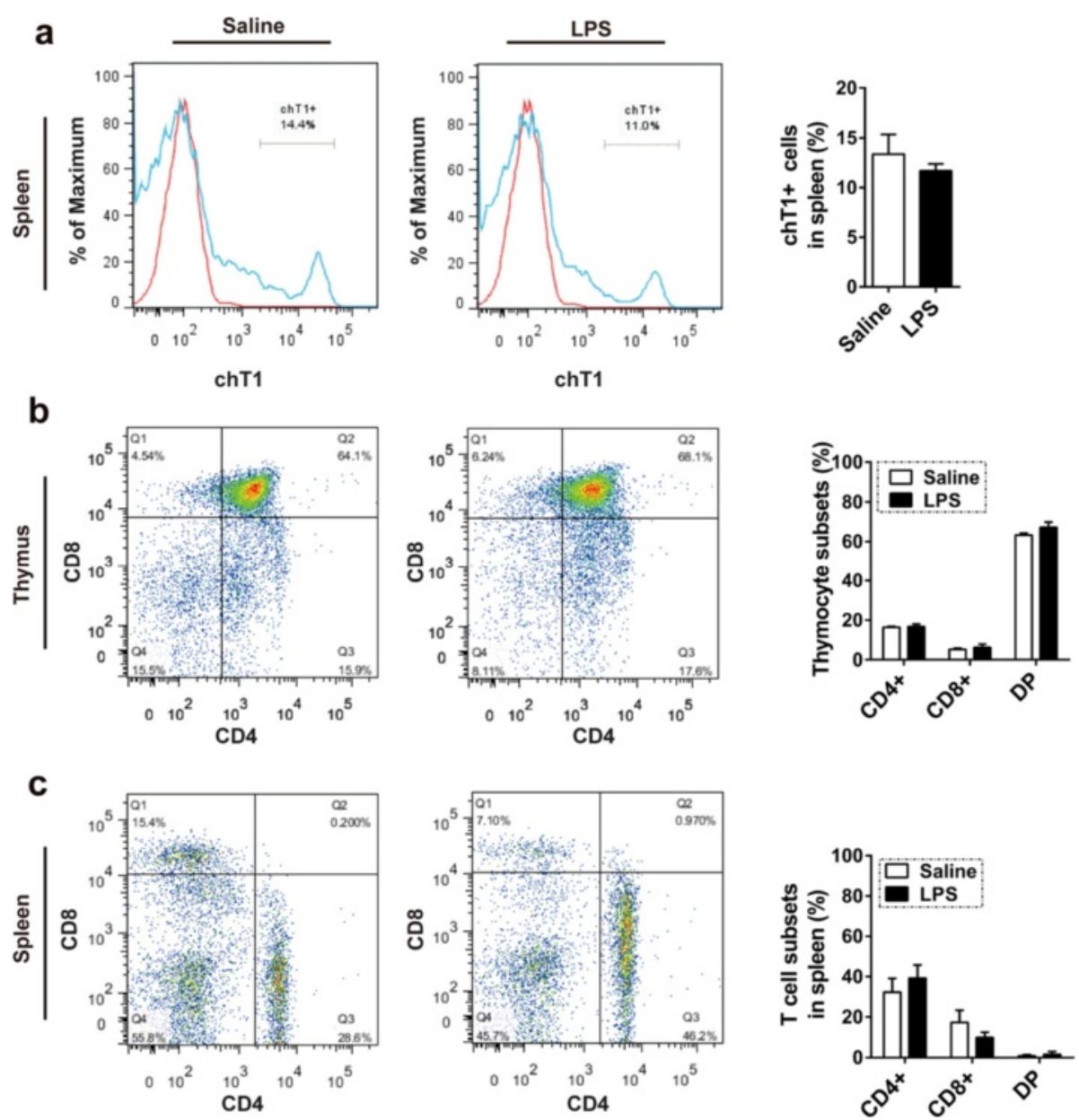

Fig. 3 Impact of Salmonella LPS on T cell population in chick thymus and spleen. Newly hatched chicks were injected i.p. with saline or 50 mg/kg Salmonella LPS and then sacrificed at 36 hpt $(n=3)$ to analyze the impact of Salmonella LPS on chick T cell populations. a chT1+ cell ratios in spleen decreased but not significantly. b, c No significant change of CD4+/CD8+ T cells in chick thymus (b) or spleen (c) was observed. All data are presented as means \pm SD. Statistically significant differences between LPS and saline groups at each time point were determined using Student's t-test

IL8L2, SOCS3, PPARG, were up-expressed in the TLR4FOS/JUN signaling pathway. Genes, including STT3A, $C D C 25 A, C D K 1 / 6, C C N A, C C N B, C C N D$ and $C C N E$, involved in the cell cycle pathway were down-expressed. Furthermore, the oxidative and calcium stress related genes of SOD and calpain were up-expressed (Fig. 6b; Additional file 1: Figure S5a; Additional file 4: Table S3). These changes were attenuated at $72 \mathrm{~h}$ after LPS treatment (Additional file 1: Figure S5b; Additional file 4: Table S3).

\section{Discussion}

The thymus is a common target for infectious diseases without exception in mammals and birds $[2,4,5]$. In the present study, we found that STm induced acute thymic injury in newly hatched chicks, which was represented by thymocyte death and organic atrophy. Previous studies also found multiple pathogens, including parasites (e.g., Ascaridia galli), virus (e.g., chicken anemia agent) and bacteria (e.g., E. coli), could induce thymic atrophy in chickens $[6,9,23]$. Therefore, thymic atrophy is a common consequence of pathogenic infection in chickens.

We found both STm and Salmonella LPS could induce thymocyte death and thymic atrophy. They also induced almost the same patterns of gene expression, cell death and organic atrophy, although LPS acted more rapidly than STm. The maximum thymocyte death and thymic atrophy occurred at 12 and $36 \mathrm{~h}$, respectively, with LPS challenge, while they occurred at 36 and $72 \mathrm{~h}$, respectively, following STm infection. LPS has been confirmed to induce inflammation through the TLR4 signaling pathway [24-26]. Therefore, we deduced that LPS may be the major contributor to the thymus injury induced by STm infection, which acts by triggering intrathymic inflammation. Given the size of LPS versus bacteria, the delayed thymic reaction following STm 


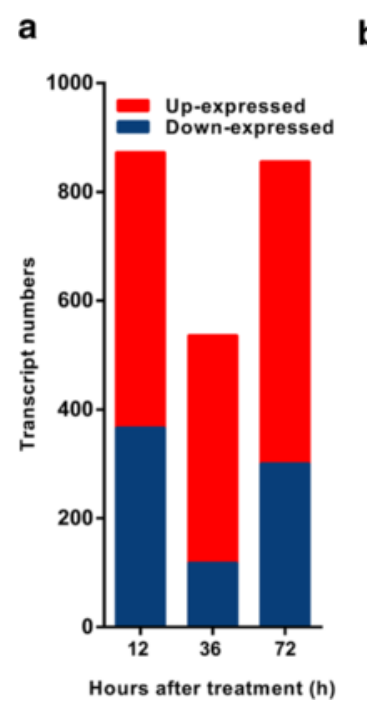

\begin{tabular}{|c|c|c|c|c|c|}
\hline \multicolumn{2}{|c|}{$12 \mathrm{~h}$} & \multicolumn{2}{|c|}{$36 \mathrm{~h}$} & \multicolumn{2}{|c|}{$72 \mathrm{~h}$} \\
\hline Gene Symbol & Fold change & Gene Symbol & Fold change & Gene Symbol & Fold change \\
\hline$A V D$ & 191.6 & $A V D$ & 61.0 & LOC101748638 & 11.1 \\
\hline AVR2 & 44.8 & $1 L 411$ & 22.8 & $C A 12$ & 11.1 \\
\hline$F G A$ & 28.2 & $F G A$ & 20.8 & LOC101752309 & 10.6 \\
\hline LOC419276 & 19.9 & 1L8L2 & 12.2 & HBE & 9.3 \\
\hline $1 L 4 / 1$ & 19.5 & STEAP4 & 10.2 & СКMT2 & 9.2 \\
\hline LOC101751347 & 16.3 & ATP13A4 & 8.6 & ATOH7 & 8.2 \\
\hline HPS5 & 15.7 & LOC101750146 & 8.2 & LOC101749796 & 7.9 \\
\hline NOV & 14.2 & LCN8 & 7.7 & GRP & 7.6 \\
\hline LCN8 & 13.2 & CMBL & 7.6 & LOC425214 & 7.3 \\
\hline 12.22 & 10.8 & CYP8B1 & 7.4 & LOC417386 & 6.7 \\
\hline EEF1A1 & -503.7 & LOC101751536 & -8.1 & $T G$ & -141.5 \\
\hline TG & -73.8 & LOC101751579 & -6.9 & $N K X 2-1$ & -20.2 \\
\hline IYD & -13.3 & THRSPB & -4.3 & IYD & -8.9 \\
\hline LOC101751579 & -8.9 & LOC101747510 & -4.2 & TULP1 & -8.2 \\
\hline LEAP2 & -8.2 & LOC101749667 & -3.3 & LOC101747727 & -8.0 \\
\hline CNTNAP5 & -7.6 & FAM183A & -3.1 & LOC430443 & -6.9 \\
\hline FOXE1 & -4.2 & $A G R P$ & -3.1 & LEAP2 & -6.3 \\
\hline LOC101750727 & -3.9 & CNTNAP5 & -3.0 & PDK4 & -5.6 \\
\hline CCL17 & -3.4 & KRT14 & -2.6 & LOC772126 & -5.5 \\
\hline SPINK5 & -3.2 & C28H19orf77 & -2.4 & LOC101749560 & -5.5 \\
\hline
\end{tabular}

Fig. 4 Analysis of DETs in chick thymus after Salmonella LPS treatment. Newly hatched chicks were injected i.p. with saline or 50 mg/kg Salmonella LPS and then sacrificed at defined time points $(n=7 \sim 9)$ to perform transcriptome analysis of the thymus. DETs were determined using the MARS model in DEGseq package between different time points (12 hpt vs. 0 hpt, 36 hpt vs. 0 hpt, 72 hpt vs. 0 hpt) with the same cut-off ( $P<0.001$, absolute fold change $\geq 1.5$ ). a Numbers of DETs at different time point. $\mathbf{b}$ Top ten up- and down-expressed genes at different time point. The positive values indicate up-expression and negative values indicate down-expression

infection may be resulted from the longer time for bacteria enter the tissue and interact with TLR4 than single LPS molecular. Besides, LPS treatment did not disrupt splenic chT1+ cells and CD4+/CD8+ T cell ratios in thymus and spleen tissues in chicks. The chT1 is an index to evaluate recent thymic export [21]. The maintenance of splenic chT1+ cells suggested that recent thymic export may not be disturbed at $36 \mathrm{~h}$ when maximum thymic atrophy occurred after LPS treatment. CD4 + CD8+ DP T cells are the main subpopulations in thymus [1]. The maintenance of DP and SP $\mathrm{T}$ cell ratios indicated that the composition of CD4+/CD8+ T cell subpopulations may not be changed at $36 \mathrm{~h}$ after LPS treatment. Our results were consistent with those of a previous study in mice infected with STm [13]. Thus, thymic dysfunction may not be inevitable with thymic injury due to pathogenic treatment.

To gain further insight into the acute chick thymic injury induced by Salmonella LPS, we performed transcriptome analysis of the thymus. We found that inflammatory cytokines, DNA damage genes and cell cycle genes were changed after LPS challenge. IPA results indicated that the acute inflammation mediated through the TLR4-FOS/JUN signaling pathway may play an important role in thymic atrophy. Based on the present studies and those findings published by other groups [24-39], we concluded that the transcriptional factors FOS/JUN were activated upon LPS binding to TLR4 protein, consequently inducing the release of inflammatory factors IL8L2 and CCL4. These inflammatory factors, including $I L-22, I L 8 L 2$ and $C C L 4$, could induce the inflammation and promote the oxidative and calcium stress. These reactions resulted in DNA damage and cell cycle arrest, which contributed to the thymic atrophy in chicks. TLR4 is a specific pattern recognition receptor for LPS in birds and mammals $[24,27]$. In mice, deficiency of TLR4 was shown to prevent thymocyte death and thymic atrophy when challenged by $E$. coli LPS $[28,29]$. Transcriptional factors FOS/JUN can form the AP-1 dimeric complex to regulate cell proliferation and death [30]. AP-1 can be activated by LPS-TLR4 signaling and then induce the expression of inflammatory cytokines, including IL8 and CCL4, which contribute to inflammation by recruiting and activating leukocytes [27, 31, 32]. LPS stimulation also has been shown to induce the expression of another inflammatory factor IL22 in vivo, and overexpression of IL22 alone could induce thymic atrophy in mice $[33,34]$. Furthermore, inflammatory reactions can promote the production of ROS [35, 36]. ROS alone can damage the host DNA, and it also can interact with calcium signaling to induce cell death and cell cycle arrest [37-39]. Thus, thymic atrophy induced by STm or Salmonella LPS in this study was considered to be mainly or at least partially mediated through the TLR4-FOS/JUN signaling pathway in chicks.

We also found that some anti-inflammatory factors, including BPI, PPARY and SOCS3, were activated after LPS stimulation. BPI can inhibit formation of the LPSTLR4 complex through competing with LPS [40]. PPAR $\gamma$ can inhibit FOS/JUN and NF- $\mathrm{KB}$ by preventing their binding to target sequences [41, 42]. SOCS3 can 


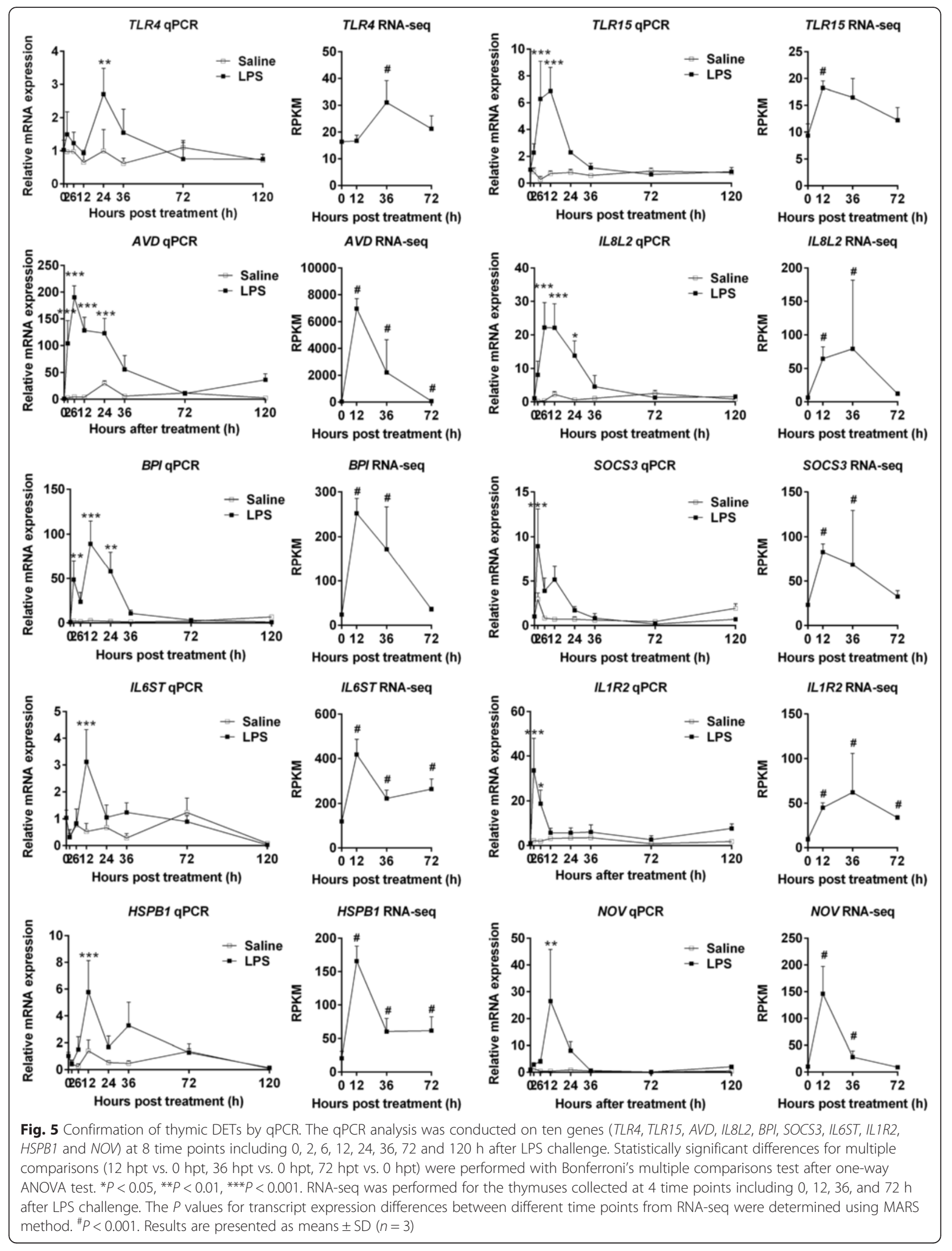




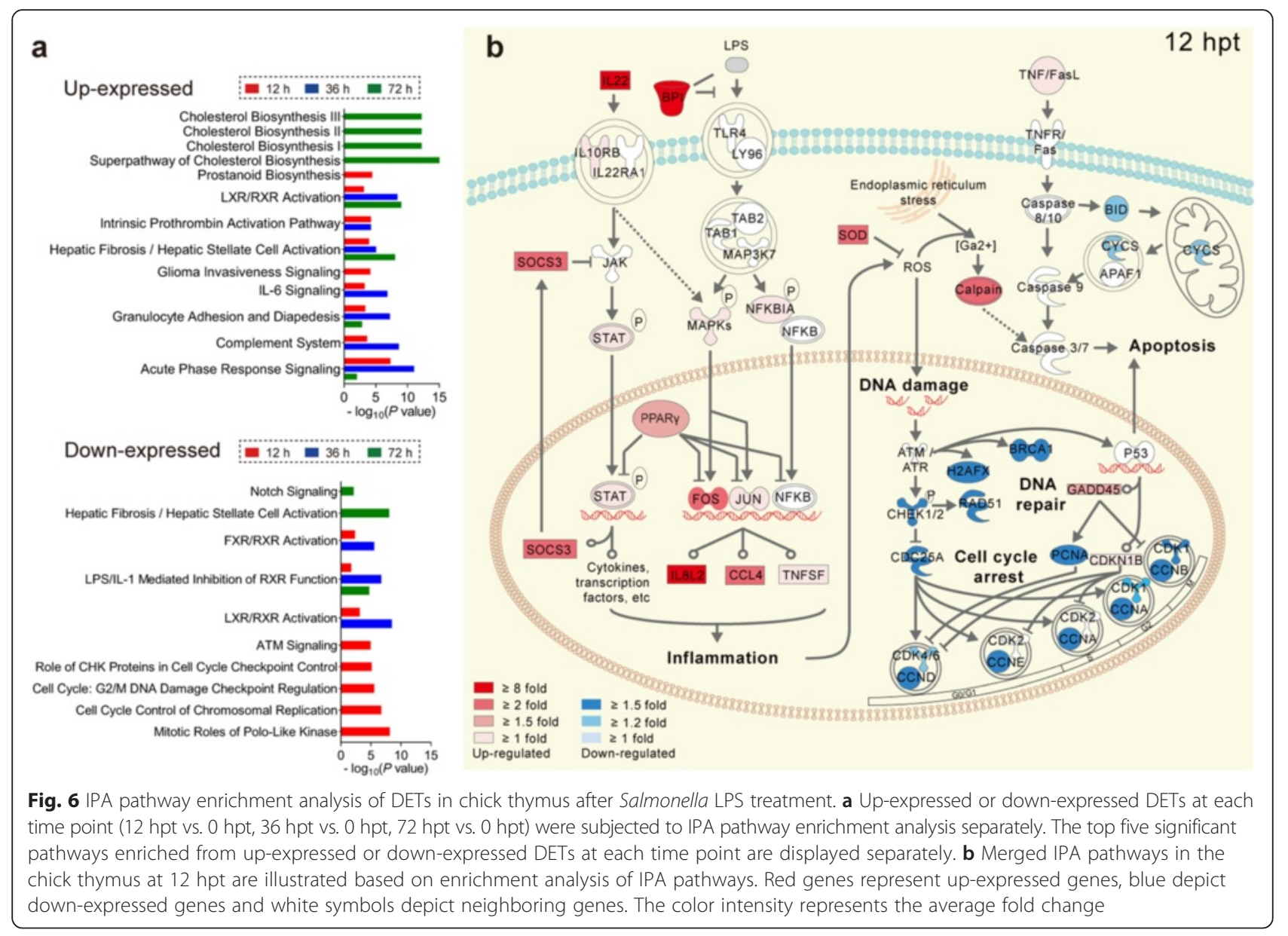

inhibit STAT phosphorylation through binding of JAK kinase [43]. Thus, negative inflammation response regulators naturally can also be activated when the host is infected by pathogens in order to maintain the immune homeostasis and avoid excessive tissue injury [44, 45]. In addition, we observed apoptosis mediated through the mitochondrial pathway at $72 \mathrm{~h}$ of LPS treatment. Moreover, the thymus weight was restored to the normal level at $120 \mathrm{~h}$. These finding indicated that compensatory growth occurred after the acute inflammatory injury. The phenomenon of compensatory growth of the thymus after injury also has been observed in mice [46].

\section{Conclusions}

We confirmed that STm and Salmonella LPS could induce thymocyte death and thymic atrophy in chicks. LPS could be the main factor in the thymic atrophy during a STm infection. Inflammatory reactions were the factor contributing to the thymic atrophy after LPS treatment in chicks. Moreover, this work associated with findings published by other groups [24-39] demonstrates that this inflammatory process may be mediated through the TLR4-FOS/JUN signaling pathway.
Our results provide novel evidence for the molecular mechanism of the thymus injury induced by LPS derived from STm.

\section{Methods}

Ethics statement

This study was approved by the Ethics Committee of Huazhong Agricultural University (HZAUMU2013-0005). The experiments were performed in accordance with the Guide for the Care and Use of Laboratory Animals (1996), and protocols were approved by the Hubei Province for Biological Studies Animal Care and Use Committee.

\section{Animals}

Healthy newly hatched broiler chicks ( $\leq 3$ days old, Cobb 500) were obtained from the Wuhan Zhengda chicken breeding company and housed under conventional conditions without any vaccinations. Chicks were injected intraperitoneally (i.p.) with $0.5 \mathrm{~mL}$ of $5 \times 10^{4} \mathrm{CFU} / \mathrm{mL}$ of live STm strain cvcc541 (China Veterinary Culture Collection Center, Beijing, China) or LPS derived from STm (L7261; Sigma-Aldrich, St. Louis, MO, USA) at $50 \mathrm{mg} /$ $\mathrm{kg}$ of body weight in $0.5 \mathrm{~mL}$ avian saline $(0.75 \% \mathrm{NaCl})$. 
The control group was injected only with $0.5 \mathrm{~mL}$ avian saline. Six chicks per treatment (STm or saline injection) per time point $(0,12,36,72$ and $120 \mathrm{~h})$ were sampled in STm infection experiment. At least four chicks per treatment (LPS or saline injection) per time point $(0,2,6,12$, 24, 36, 72 and $120 \mathrm{~h}$ ) were sampled for morphology and qPCR analyses. For transcriptome analysis of chick thymus after LPS treatment, at least seven chicks per time point $(0,12,36$ and $72 \mathrm{~h})$ were sampled. Thymuses were collected and weighed at defined time points. The thymus index was calculated using the formula: Thymus index $(\mathrm{mg} / \mathrm{g})$ = weight of thymus $(\mathrm{mg}) /$ body weight $(\mathrm{g})$ [47]. For morphological analysis, fresh thymuses were fixed in $4 \%$ paraformaldehyde, embedded into paraffin and stored at $4{ }^{\circ} \mathrm{C}$. For gene expression analysis, fresh thymuses were frozen in liquid nitrogen and stored at $-70{ }^{\circ} \mathrm{C}$. For cell subpopulation analysis, fresh thymuses and spleens were collected and performed flow cytometric assays without storing. All organ sampling for each chick was finished in thirty minutes.

\section{Hematoxylin and eosin staining}

Thymic tissues embedded in paraffin were cut into 4$\mu \mathrm{m}$-thick sections. The sections were then deparaffinized in xylene, rehydrated in a graded series of alcohol to water. Slides were stained with Harris hematoxylin solution for $5 \mathrm{~min}$ and differentiated in acid alcohol for $10 \mathrm{~s}$. After rinsing in running tap water for $15 \mathrm{~min}$, the sections were then stained in eosin solution for $1 \mathrm{~min}$, dehydrated in alcohol, cleared in xylene and mounted with coverslips. Stained samples were examined by light microscopy (Olympus BX51, Tokyo, Japan) with a digital camera (DP72; Olympus).

\section{Formamide-monoclonal antibody (MAb) assay}

The rehydrated thymic sections were incubated with $20 \mu \mathrm{g} / \mathrm{ml}$ proteinase $\mathrm{K}$ and $0.1 \mathrm{mg} / \mathrm{ml}$ saponin in PBS for $20 \mathrm{~min}$ at room temperature (RT) and then incubated in $50 \%(\mathrm{v} / \mathrm{v})$ formamide in distilled water at $56{ }^{\circ} \mathrm{C}$ for $20 \mathrm{~min}$ before transferring to cold PBS for $5 \mathrm{~min}$. The sections were then incubated with a primary mouse IgM anti-ssDNA MAb (clone F7-26; EMD Millipore, Billerica, MA, USA) at a dilution of $1: 10$ for $30 \mathrm{~min}$ at $37^{\circ} \mathrm{C}$. Secondary antibody incubation was performed using the antimouse IgM SABC kit (Boster, Wuhan, China). After visualization with $\mathrm{DAB}$ solution, the sections were counterstained with hematoxylin. Sections were examined by light microscopy (Olympus BX51) with a digital camera (DP72; Olympus). Sections were sampled by random movement of the mechanical microscope stage to bring new, non-overlapping areas (at least 5 fields) into view. Counts were standardized from the mean number of formamide-MAb-positive cells per field to the number per $1 \times 10^{5} \mu \mathrm{m}^{2}$.

\section{TUNEL assay}

The TUNEL assay was performed using the In Situ Cell Death Detection Kit, POD (Roche Boehringer Mannheim Corp., Indianapolis, IN, USA) according to the manufacturer's instruction. The rehydrated thymic sections were incubated with terminal deoxynucleotidyl transferase $(\mathrm{TdT})$ in a reaction buffer for $1 \mathrm{~h}$ at $37^{\circ} \mathrm{C}$. Thereafter, the sections were washed in PBS and observed using a fluorescence microscope (Olympus, Tokyo, Japan) with a digital camera (DP72; Olympus) before the following steps were performed. Endogenous peroxides were blocked by incubation in $3 \% \mathrm{H}_{2} \mathrm{O}_{2}$ in PBS for $10 \mathrm{~min}$ at RT. Fluorescent signal conversion was performed using an anti-fluorescein antibody conjugated with peroxides. Sections were incubated with DAB solution and counterstained with hematoxylin. Cell counting and statistical analysis for TUNEL-positive cells were conducted in the same manner as that for formamideMAb-positive cells mentioned above.

\section{Flow cytometry}

Thymus and spleen tissues were prepared to obtain single-cell suspensions $\left(2 \times 10^{6}\right.$ cells $)$ as previously described [48]. Immunofluorescence staining was performed with anti-chicken directly-conjugated MAbs: anti-CD4 FITC, anti-CD8 PE and anti-chT1 FITC (AbD Serotec, Ltd., Oxford, UK). Cell suspensions were added to PBS wash buffer containing $2 \%$ bovine serum albumin and diluted antibodies for $30 \mathrm{~min}$ at $4{ }^{\circ} \mathrm{C}$, followed by washing and resuspension in PBS containing $1 \%$ paraformaldehyde. All cell populations were assessed using a FACSAria III (BD Biosciences, San Jose, CA, USA).

\section{RNA-seq and data analysis}

Total RNA was isolated from each thymic sample using the standard TRIzol protocol (Invitrogen, Carlsbad, CA, USA). RNA quality was examined by gel electrophoresis and with a Nanodrop spectrophotometer (Thermo, Waltham, MA, USA). For RNA sequencing, RNA samples from seven to nine biological replicates at each time point $(0,12,36$ and $72 \mathrm{~h})$ were separated into three independent pools, each comprised of two or three distinct samples, at equal amounts. Strand-specific libraries were constructed using the TruSeq RNA sample preparation kit (Illumina, San Diego, CA, USA), and sequencing was carried out using the Illumina HiSeq 2000 instrument by the commercial service of Genergy Biotechnology Co. Ltd. (Shanghai, China). The raw data was handled by Perl and data quality was checked by FastQC 0.11.2 (http:// www.bioinformatics.babraham.ac.uk/projects/fastqc/). The read length was $50 \mathrm{bp}$. Clean reads were aligned to the chicken genome (release: Gallus gallus 4.0) from NCBI using Bowtie [49], with one mismatch allowed. Genome mapped data were annotated using the gff 3 file of the 
Gallus gallus 4.0 genome (ftp://ftp.ncbi.nlm.nih.gov/genomes/Gallus_gallus/). The expression of the transcript was calculated by RPKM (Reads Per Kilobase of exon model per Million mapped reads) using Perl. Differentially expression transcripts (DETs) were determined using the MA-plot-based method with Random Sampling (MARS) model in the DEGseq package between different time points (12 hpt vs. $0 \mathrm{hpt}, 36 \mathrm{hpt}$ vs. $0 \mathrm{hpt}$, $72 \mathrm{hpt}$ vs. $0 \mathrm{hpt}$ ) [50]. Generally, in MARS model, $M=$ $\log _{2} C_{1}-\log _{2} C_{2}$, and $A=\left(\log _{2} C_{1}+\log _{2} C_{2}\right) / 2\left(C_{1}\right.$ and $C_{2}$ denote the counts of reads mapped to a specific gene obtained from two samples) [50]. The thresholds for determining DETs are $P<0.001$ and absolute fold change $\geq 1.5$. Then DETs were chosen for signaling pathway enrichment analysis using Ingenuity Pathway Analysis (IPA) software. The significantly enriched pathways were determined when $P<0.05$ and at least two affiliated genes were included.

\section{Quantitative real-time PCR (qPCR)}

Total RNA was isolated from thymuses of three chicks at each time point $(0,2,6,12,24,36,72$ and $120 \mathrm{~h})$ of LPS treatment group or saline control group. In addition, RNA was also isolated from thymuses of three chicks at each time point $(0,12,36,72$ and $120 \mathrm{~h})$ under STm infection. Then total RNA were treated with RNase-free DNase I (Fermentas, Opelstrasse, Germany) to remove contaminating genomic DNA. The first strand cDNA was synthesized using the RevertAid First Strand cDNA Synthesis Kit (Fermentas, Opelstrasse, Germany). The reaction mixture $(10 \mu \mathrm{l})$ for qPCR contained of $5 \mu \mathrm{L}$ SYBR Select Master Mix for CFX (Applied Biosystems), $0.2 \mu \mathrm{L}$ of each forward and reverse primer and $1 \mu \mathrm{L}$ of template cDNA. The qPCR reactions were performed on a Bio-Rad CFX Connect real-time PCR detection system (Bio-Rad, Hercules, CA, USA). The qPCR conditions were as follows: pre-denaturation at $95{ }^{\circ} \mathrm{C}$ for $5 \mathrm{~min}$, followed by 40 cycles of denaturation at $95{ }^{\circ} \mathrm{C}$ for $30 \mathrm{~s}$, annealing at $60{ }^{\circ} \mathrm{C}$ for $30 \mathrm{~s}$, and elongation at $72{ }^{\circ} \mathrm{C}$ for $20 \mathrm{~s}$. The primer sequences were listed in Additional file 5: Table S4. ACTB was chosen as a reference for qPCR. All samples were run in triplicate and gene expression levels were quantified using the $\Delta \Delta \mathrm{Ct}$ method [51].

\section{Statistical analysis}

Data were calculated as the means \pm standard deviation (SD). All analyses and graphic representations were performed with Prism software 5.01 (GraphPad Software, Inc., San Diego, USA). The statistical significance in mean values between two-group comparison was performed using two-tailed Student's $t$-test (Fig. 1a and b; Fig. 2; Fig. 3; Additional file 1: Figure S1). The statistical significance in the comparison of multiple sample sets versus control was performed with Bonferroni's multiple comparisons test after one-way ANOVA test
(Fig. 1c and d; Fig. 5). The correlation analysis between changes of ten genes detected by qPCR and RNA-seq was performed and the significance was detected using Pearson's test (Additional file 1: Figure S2). Differences were considered significant if $P<0.05$. ${ }^{*} P<0.05$, ${ }^{* *} P<$ 0.01 and $* \% P<0.001$.

\section{Availability of supporting data}

All the datasets supporting the results have been listed in the article and its additional files. The RNA-seq data has been deposited to the National Center for Biotechnology Information (NCBI) Short Read Archive (SRA) under accession code SRP065372.

\section{Additional files}

Additional file 1: Supplementary figures. (PDF $1259 \mathrm{~kb}$ )

Additional file 2: Table S1. Detailed information on DETs at different time points. a Up-expressed DETs at $12 \mathrm{hpt}$. b Down-expressed DETs at $12 \mathrm{hpt}$. c Up-expressed DETs at $36 \mathrm{hpt}$. d Down-expressed DETs at 36 hpt. e Up-expressed DETs at $72 \mathrm{hpt}$. f Down-expressed DETs at $72 \mathrm{hpt}$. Transcripts were considered as DETs only with absolute fold changes $\geq$ 1.5 and $P<0.001$. (XLS $387 \mathrm{~kb}$ )

Additional file 3: Table S2. Detailed information on IPA pathway analysis of DETs at different time points. a Over-represented pathways from up-expressed DETs at $12 \mathrm{hpt}$. b Over-represented pathways from down-expressed DETs at $12 \mathrm{hpt}$. c Over-represented pathways from up-expressed DETs at $36 \mathrm{hpt}$. d Over-represented pathways from down-expressed DETs at $36 \mathrm{hpt}$. e Over-represented pathways from up-expressed DETs at 72 hpt. f Over-represented pathways from down-expressed DETs. IPA pathways with $P<0.05$ and at least two genes affiliated were reported in the present study. (XLS 63 kb)

Additional file 4: Table S3. Detailed information on genes involved in merged IPA pathways at different time point. Genes involved in merged IPA pathways with $P<0.001$ were considered. Dashes indicated no significant difference in the genes expression. (XLS $21 \mathrm{~kb}$ )

Additional file 5: Table S4. List of qPCR primers. (XLS $27 \mathrm{~kb}$ )

\section{Competing interests}

The authors declare that they have no competing interests.

\section{Authors' contributions}

This study was conceived and designed by $\mathrm{HL}$ and $\mathrm{HH}$. HH performed the experiments and data analysis, and prepared the manuscript. AL contributed to the analysis of RNA-seq data. HW contributed to the qPCR experiment. AA and JW contributed to experimental animals' treatment and histopathologic staining. $\mathrm{XH}$ and $\mathrm{XZ}$ contributed to sample collection and flow cytometry experiment. $\mathrm{HL}, \mathrm{JZ}$ and PK participated in the interpretation of the results and finalized the manuscript. All authors reviewed the manuscript and agreed with its publication.

\section{Acknowledgements}

We thank Anna Du and Juan Min from the State Key Laboratory of Virology (Wuhan Institute of Virology) for their excellent technical assistance.

\section{Funding}

This work was supported by grants from the National Natural Science Foundation of China (30800808), and the Fundamental Research Funds for the Central Universities (2012ZYTS048, 2013YB09, 2014PY046).

\section{Author details}

${ }^{1}$ Department of Basic Veterinary Medicine, College of Animal Science and Veterinary Medicine, Huazhong Agricultural University, Wuhan 430070, China. ${ }^{2}$ Key Lab of Animal Genetics, Breeding and Reproduction of Ministry 
Education, College of Animal Science and Technology, Huazhong Agricultural University, Wuhan 430070, China. ${ }^{3}$ Department of Anatomy, Physiology and Pharmacology, College of Veterinary Medicine, Auburn University, Auburn, AL 36849, USA.

\section{Received: 21 December 2015 Accepted: 26 April 2016 Published online: 04 May 2016}

\section{References}

1. Gameiro J, Nagib P, Verinaud L. The thymus microenvironment in regulating thymocyte differentiation. Cell Adh Migr. 2010;4(3):382-90.

2. Raviola E, Karnovsky MJ. Evidence for a blood-thymus barrier using electronopaque tracers. J Exp Med. 1972:136(3):466-98.

3. Savino $\mathbf{W}$. The thymus is a common target organ in infectious diseases. PLoS Pathog. 2006;2(6):e62.

4. Nunes-Alves C, Nobrega C, Behar SM, Correia-Neves M. Tolerance has its limits: how the thymus copes with infection. Trends Immunol. 2013;34(10):502-10.

5. Hoerr FJ. Clinical aspects of immunosuppression in poultry. Avian Dis. 2010; 54(1):2-15.

6. Nakamura K, Imada Y, Maeda M. Lymphocytic depletion of bursa of Fabricius and thymus in chickens inoculated with Escherichia coli. Vet Pathol. 1986:23(6):712-7.

7. Nakamura K, Yuasa N, Abe H, Narita M. Effect of infectious bursal disease virus on infections produced by Escherichia coli of high and low virulence in chickens. Avian Pathol. 1990;19(4):713-21.

8. Ramadan HH, Abou Znada NY. Some pathological and biochemical studies on experimental ascaridiasis in chickens. Nahrung. 1991;35(1):71-84.

9. Sadun EH. Studies on the pathogenicity in chickens of single infections of variable size with the Nematode, Ascaridia Galli. Poult Sci. 1950;29(5):712-22.

10. Newell DG, Koopmans M, Verhoef L, Duizer E, Aidara-Kane A, Sprong H, Opsteegh M, Langelaar M, Threfall J, Scheutz F. Food-borne diseases - the challenges of 20 years ago still persist while new ones continue to emerge. Int J Food Microbiol. 2010;139 Suppl 1:S3-15.

11. Barrow PA. The paratyphoid salmonellae. Rev Sci Tech. 2000;19(2):351-75.

12. Deobagkar-Lele M, Victor ES, Nandi D. c-Jun NH2 -terminal kinase is a critical node in the death of CD4+ CD8+ thymocytes during Salmonella enterica serovar Typhimurium infection. Eur J Immunol. 2014:44(1):137-49.

13. Ross EA, Coughlan RE, Flores-Langarica A, Lax S, Nicholson J, Desanti GE, Marshall $J$, Bobat $\mathrm{S}$, Hitchcock J, White A. Thymic function is maintained during Salmonella-induced atrophy and recovery. J Immunol. 2012;189(9):4266-74.

14. Withanage GS, Kaiser $P$, Wigley P, Powers $C$, Mastroeni $P$, Brooks $H$, Barrow P, Smith A, Maskell D, McConnell I. Rapid expression of chemokines and proinflammatory cytokines in newly hatched chickens infected with Salmonella enterica serovar typhimurium. Infect Immun. 2004;72(4):2152-9.

15. Withanage GS, Wigley P, Kaiser P, Mastroeni P, Brooks H, Powers C, Beal R, Barrow P, Maskell D, McConnell I. Cytokine and chemokine responses associated with clearance of a primary Salmonella enterica serovar Typhimurium infection in the chicken and in protective immunity to rechallenge. Infect Immun. 2005;73(8):5173-82.

16. Martin SJ, Green DR. Protease activation during apoptosis: death by a thousand cuts? Cell. 1995;82(3):349-52.

17. Frankfurt OS, Robb JA, Sugarbaker EV, Villa L. Monoclonal antibody to single-stranded DNA is a specific and sensitive cellular marker of apoptosis. Exp Cell Res. 1996;226(2):387-97.

18. Frankfurt OS, Robb JA, Sugarbaker EV, Villa L. Apoptosis in breast carcinomas detected with monoclonal antibody to single-stranded DNA: relation to bcl2 expression, hormone receptors, and lymph node metastases. Clin Cancer Res. 1997;3(3):465-71.

19. Grasl-Kraupp B, Ruttkay-Nedecky B, Koudelka H, Bukowska K, Bursch W, Schulte-Hermann R. In situ detection of fragmented DNA (TUNEL assay) fails to discriminate among apoptosis, necrosis, and autolytic cell death: a cautionary note. Hepatology. 1995;21(5):1465-8.

20. Katevuo K, Imhof BA, Boyd R, Chidgey A, Bean A, Dunon D, Gobel TW, Vainio O. ChT1, an Ig superfamily molecule required for $\mathrm{T}$ cell differentiation. J Immunol. 1999;162(10):5685-94

21. Kong $\mathrm{F}$, Chen $\mathrm{CH}$, Cooper MD. Thymic function can be accurately monitored by the level of recent T cell emigrants in the circulation. Immunity. 1998:8(1):97-104.

22. Kong FK, Chen CL, Six A, Hockett RD, Cooper MD. T cell receptor gene deletion circles identify recent thymic emigrants in the peripheral T cell pool. Proc Natl Acad Sci U S A. 1999;96(4):1536-40
23. Jeurissen SH, Pol JM, de Boer GF. Transient depletion of cortical thymocytes induced by chicken anaemia agent. Thymus. 1989:14(1-3):115-23.

24. Brownlie R, Allan B. Avian toll-like receptors. Cell Tissue Res. 2011;343(1):121-30.

25. Lu YC, Yeh WC, Ohashi PS. LPS/TLR4 signal transduction pathway. Cytokine. 2008:42(2):145-51.

26. Hoshino K, Takeuchi O, Kawai T, Sanjo H, Ogawa T, Takeda Y, Takeda K, Akira S. Cutting edge: Toll-like receptor 4 (TLR4)-deficient mice are hyporesponsive to lipopolysaccharide: evidence for TLR4 as the Lps gene product. J Immunol. 1999:162(7):3749-52

27. Akira S, Takeda K. Toll-like receptor signalling. Nat Rev Immunol. 2004;4(7):499-511.

28. Wang SD, Huang KJ, Lin YS, Lei HY. Sepsis-induced apoptosis of the thymocytes in mice. J Immunol. 1994;152(10):5014-21.

29. Norimatsu M, Ono T, Aoki A, Ohishi K, Tamura Y. In-vivo induction of apoptosis in murine lymphocytes by bacterial lipopolysaccharides. J Med Microbiol. 1995:43(4):251-7.

30. Shaulian E, Karin M. AP-1 as a regulator of cell life and death. Nat Cell Biol. 2002;4(5):E131-6.

31. Li X, Jiang S, Tapping RI. Toll-like receptor signaling in cell proliferation and survival. Cytokine. 2010;49(1):1-9.

32. Ono SJ, Nakamura T, Miyazaki D, Ohbayashi M, Dawson M, Toda M. Chemokines: roles in leukocyte development, trafficking, and effector function. J Allergy Clin Immunol. 2003:111(6):1185-99. quiz 1200.

33. Liang SC, Nickerson-Nutter C, Pittman DD, Carrier Y, Goodwin DG, Shields KM, Lambert AJ, Schelling SH, Medley QG, Ma HL et al. IL-22 induces an acutephase response. J Immunol. 2010;185(9):5531-8.

34. Weber GF, Schlautkotter S, Kaiser-Moore S, Altmayr F, Holzmann B, Weighardt H. Inhibition of interleukin-22 attenuates bacterial load and organ failure during acute polymicrobial sepsis. Infect Immun. 2007;75(4):1690-7.

35. Mittal M, Siddiqui MR, Tran K, Reddy SP, Malik AB. Reactive oxygen species in inflammation and tissue injury. Antioxid Redox Signal. 2014;20(7):1126-67.

36. Hakim J. Reactive oxygen species and inflammation. C R Seances Soc Biol Fil. 1993;187(3):286-95.

37. Forman $\mathrm{HJ}$, Torres $\mathrm{M}$. Reactive oxygen species and cell signaling: respiratory burst in macrophage signaling. Am J Respir Crit Care Med. 2002;166(12 Pt 2):S4-8.

38. Nathan C, Cunningham-Bussel A. Beyond oxidative stress: an immunologist's guide to reactive oxygen species. Nat Rev Immunol. 2013;13(5):349-61.

39. Harada A, Sekido N, Akahoshi T, Wada T, Mukaida N, Matsushima K. Essential involvement of interleukin-8 (IL-8) in acute inflammation. J Leukoc Biol. 1994; 56(5):559-64.

40. Weiss J. Bactericidal/permeability-increasing protein (BPI) and lipopolysaccharidebinding protein (LBP): structure, function and regulation in host defence against Gram-negative bacteria. Biochem Soc Trans. 2003;31(Pt 4):785-90.

41. Clark RB. The role of PPARs in inflammation and immunity. J Leukoc Biol. 2002:71(3):388-400.

42. Chinetti G, Fruchart JC, Staels B. Peroxisome proliferator-activated receptors (PPARs): nuclear receptors at the crossroads between lipid metabolism and inflammation. Inflamm Res. 2000;49(10):497-505.

43. Carow B, Rottenberg ME. SOCS3, a major regulator of infection and inflammation. Front Immunol. 2014;5:58

44. Yoshimura A, Suzuki M, Sakaguchi R, Hanada T, Yasukawa H. SOCS, inflammation, and autoimmunity. Front Immunol. 2012;3:20.

45. Matarese G, La Cava A. The intricate interface between immune system and metabolism. Trends Immunol. 2004;25(4):193-200.

46. Hick RW, Gruver AL, Ventevogel MS, Haynes BF, Sempowski GD. Leptin selectively augments thymopoiesis in leptin deficiency and lipopolysaccharide-induced thymic atrophy. J Immunol. 2006;177(1):169-76.

47. Sharma JM, Dohms JE, Metz AL. Comparative pathogenesis of serotype 1 and variant serotype 1 isolates of infectious bursal disease virus and their effect on humoral and cellular immune competence of specific-pathogenfree chickens. Avian Dis. 1989;33(1):112-24.

48. Billard MJ, Gruver AL, Sempowski GD. Acute endotoxin-induced thymic atrophy is characterized by intrathymic inflammatory and wound healing responses. PLoS One. 2011;6(3):e17940.

49. Langmead B, Trapnell C, Pop M, Salzberg SL. Ultrafast and memory-efficient alignment of short DNA sequences to the human genome. Genome Biol. 2009:10(3):R25

50. Wang L, Feng Z, Wang X, Wang X, Zhang X. DEGseq: an R package for identifying differentially expressed genes from RNA-seq data. Bioinformatics. 2010;26(1):136-8.

51. Livak KJ, Schmittgen TD. Analysis of relative gene expression data using real-time quantitative PCR and the 2(-Delta Delta C(T)) Method. Methods. 2001;25(4):402-8. 